\title{
LABORATORY OBSERVATIONS OF IMPACTS ON COARSE SEDIMENT BEACHES
}

\author{
Ian Ball ${ }^{1}$, Edgar Mendoza-Baldwin ${ }^{2}$, David Simmonds ${ }^{1}$, Adrián Pedrozo-Acuña ${ }^{2}$ and Dominic \\ Reeve $^{1}$
}

\begin{abstract}
In this paper we present laboratory observations of plunging wave breaker impact pressure responses on a steep coarse-grained beach, extending previous work conducted by Pedrozo-Acuña et al. (2008). Scale laboratory measurements of plunging breaker impact events are reported and compared with the previous full-scale tests. These tests extend the previous relationships to a wider range of surf-similarity parameters and indicate a continued reduction in impact pressure as the transition from plunging impacts to surging impacts is approached. Additional results from scale tests conducted on a smooth impermeable slope also indicate the presence of a maximum impact pressure within the plunging breaker region; however also suggest it may be necessary to include roughness and permeability in the parameterization of the impact pressure.
\end{abstract}

Keywords: gravel beach; wave impact; plunging breaker; pressure

\section{INTRODUCTION}

Gravel beaches are commonplace across much of North America and Northern Europe and bear a large responsibility for the primary defense to sections of the coastline and hinterland that have high economic, environmental or social value (Mason and Coates, 2001). Accurate prediction of the response of these coastal environments to wave action will allow more efficient and economic management of this defence than is presently possible.

Sedimentary profile evolution of gravel beaches during wave breaking and subsequent uprush can be predicted by a number of numerical models with reasonable accuracy. Pedrozo-Acuña et al. (2006) have demonstrated good agreement between numerical prediction of profile response above the still water level. However, below the still water line such models currently are unable to predict this excavated trough. Figure 1 shows this schematically.

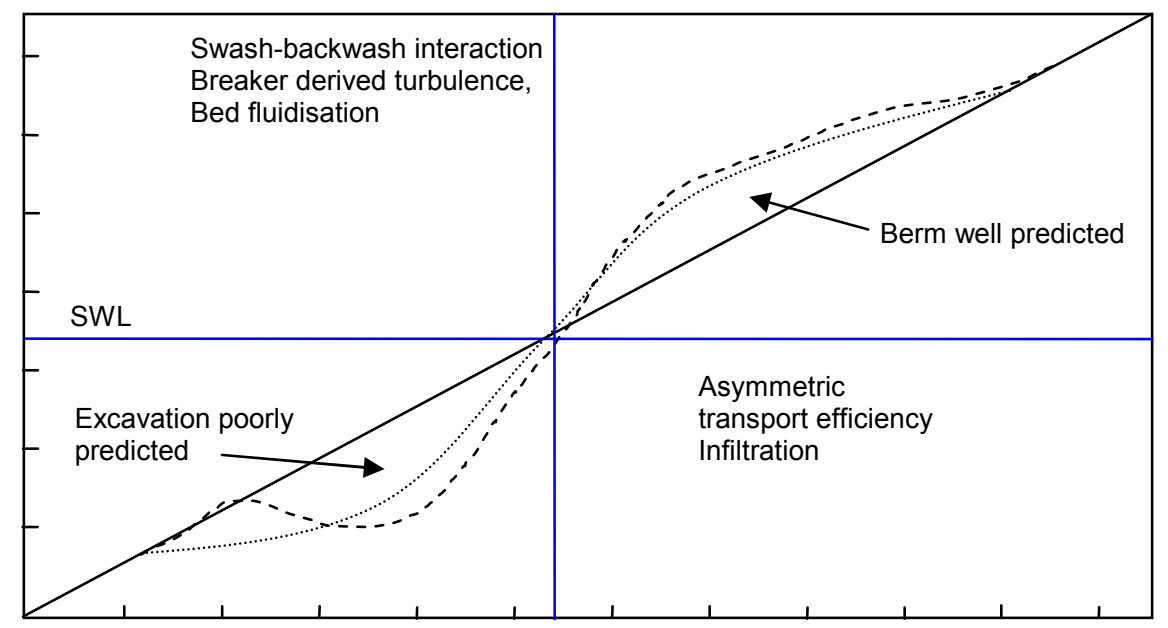

Figure 1. Schematic diagram indicating regions of good and poor model performance (after Pedrozo-Acuña et al, 2006) showing actual (dashed line) and predicted profiles (dotted line)

Whilst Pedrozo et al's model provided a good prediction of the berm size and position, there was a significant disparity in the extent, depth and shape of the excavated region. Pedrozo-Acuña indicated that the differences in profiles were related to infiltration above the beachface and the accelerated flow that occurs during uprush. Further investigation by Pedrozo-Acuña et al. (2007) extended this work through a parametric sensitivity analysis, which indicated that the accelerated flow in the uprush was insufficient to account for the observed profile differences and suggested it may be necessary to consider the additional pressures induced by wave impacts from plunging breakers.

\footnotetext{
${ }^{1}$ Coastal Engineering Research Group, University of Plymouth, Drake Circus, Plymouth, Devon, PL4 8AA, UK

${ }^{2}$ Instituto de Ingeniería, Universidad Nacional Autónoma de México, Ciudad Universitaria, Coyoacán, D.F., 04510, Mexico
} 
More recently work Jamal et al. (2010) has shown that more accurate prediction of the berm is possible; however there still exists difficulty in reproducing the profile features below the still water level. This is largely due to the absence of additional processes within the model, including sediment fluidisation, swash interaction, and the additional pressures induced by plunging breaker impacts.

Bullock et al. (2007) investigated the pressure response under plunging impacts on vertical and sloping walls and a characteristic "church-roof" profile was identified for plunging wave conditions, as schematized in Figure 2.

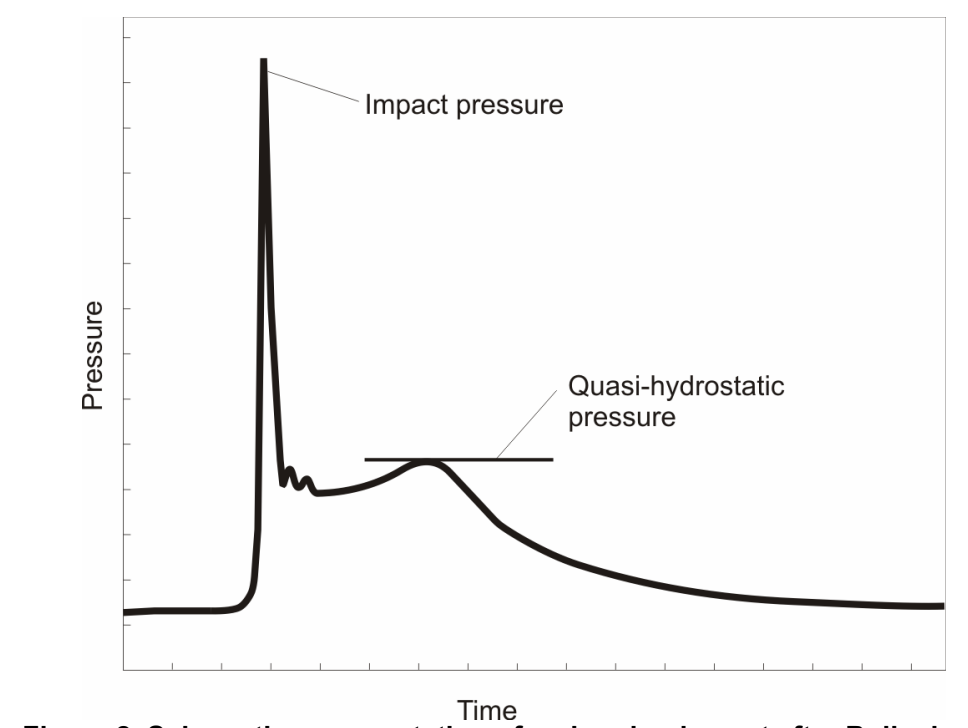

Figure 2. Schematic representation of a plunging impact after Bullock et al. (2007)

The characteristic sharp localised pressure spike is associated with the initial plunging impact, while the subsequent quasi-hydrostatic pressure is associated with the lens of water passing over the pressure transducer.

This pressure response has since been observed to occur in gravel beaches during full-scale testing (Pedrozo-Acuña et al., 2008). The data used was collected at the GWK facility in Hannover. The core of the beach was an impermeable concrete slope over which a $2 \mathrm{~m}$ layer of gavel $\left(\mathrm{D}_{50}=21 \mathrm{~mm}\right)$ was placed to form a beach with 1:7 slope. An array of pressure transducers collected pressure records at the impact location, while a wave gauge close to the breaker location provided the breaking wave conditions.

Three different test conditions were examined in the previous work for wave steepnesses $0.03-0.05$ and wave heights of the order of $1.0 \mathrm{~m}$.

The resulting impact events, identified from the time series were then studied in relationship to several parameters. A monotonic increasing trend was observed between the impact pressure of individual events non-dimensionalised by the mean quasi-hydrostatic pressure, $\rho . g . H_{s}$, and the incident wave height normalised by significant wave height, $H_{s}$. A similar trend was observed between the nondimensional impact pressures and the normalised maximum surface displacement. For both, the high degree of scatter in the data indicated an unsatisfactory parameterisation.

A further parameterization was examined in terms of the non-dimensional impact pressure and the breaker type represented by the surf-similarity number at breaking after Iribarren and Nogales (1949, equation 1).

$$
\xi_{b}=\frac{\tan \beta}{\sqrt{H_{b} / L_{0}}}
$$

Here $\beta$ is the beach slope, $H_{b}$ is the wave height at breaking point, and $L_{0}$ is the offshore wave length calculate from linear wave theory. Battjes (1974) first indicated that this number could be used to classify the breaker type for beaches with slopes between $\beta=1 / 5$ and $\beta=1 / 20$. For plunging breakers this number falls between 0.4 and 2.0.

Figure 3 shows the non-dimensional impact pressure plotted against the Iribarren number. An inverse relationship is evident. This was attributed to an increase in the steepness of the wave resulting 
in a shift towards the surging breaker regime and away from the more intense plunging breakers. However, it is clear that the majority of data falls between $0.5<\xi_{\mathrm{b}}<1.0$, i.e. in the plunging regime, and this limitation has constrained a more thorough understanding of the relationship that may exist. The aim of this work is therefore to conduct additional testing to extend the range of impact events to an area of the parameter-space not previously investigated.

From Figure 3 it might be anticipated that at larger values of surf-similarity the non-dimensional impact pressure will continue to decrease as the wave breaking moves from plunging to surging breaker type. For $\xi_{\mathrm{b}}<0.5$ it would be expected that the impact pressures would reduce by virtue of the transition from plunging to spilling breakers. Therefore a maximum in the impact pressure would be anticipated within the range $0.4<\xi_{\mathrm{b}}<2.0$.

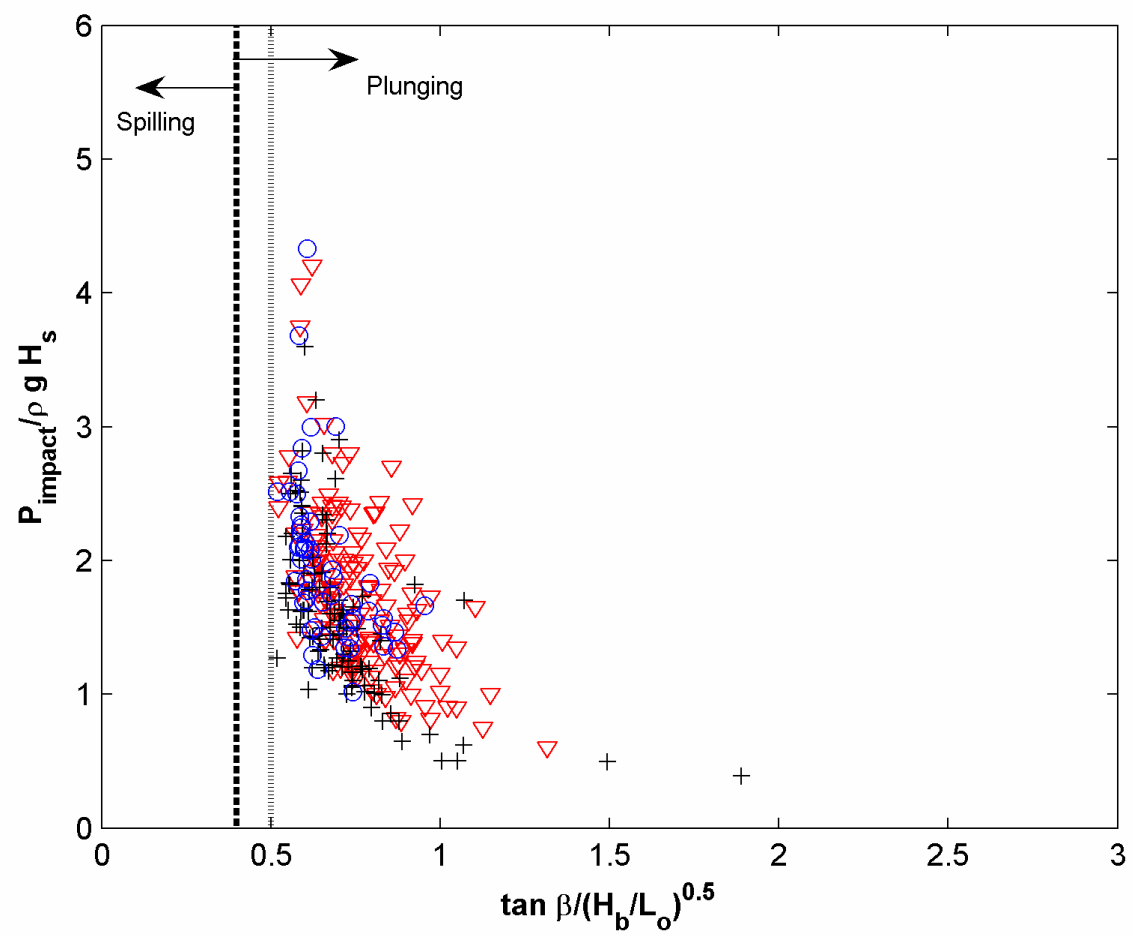

Figure 3. Non-dimensional impact pressure vs surf-similarity parameter (black cross $+H_{s}=1 \mathrm{~m} T_{p}=4.1 \mathrm{~s}$, blue circle $\circ H_{s}=1.2 \mathrm{~m} T_{p}=4.2 \mathrm{~s}$, red triangle $\nabla H_{s}=1 \mathrm{~m} T_{p}=5.2 \mathrm{~s}$ ) (using data from Pedrozo-Acuña et al., 2008)

\section{PHYSICAL EXPERIMENTATION}

The following section presents a summary of the experiments conducted at Universidad Nacional Autónoma de México (UNAM). The experiments were conducted in a $37.0 \mathrm{~m}$ long, $0.8 \mathrm{~m}$ wide and $1.2 \mathrm{~m}$ deep flume, capable of generating wave heights of $0.3 \mathrm{~m}$ and wave periods between $0.2 \mathrm{~s}$ and $4 \mathrm{~s}$.

A beach was constructed of $6 \mathrm{~mm}$ gravel forming a 1 in 5 slope. An array of pressure transducers was located within the beach to provide a constant record across the region of breaker impacts, whilst an array of wage gauges was located at the toe of the beach to record the incident and reflected wave conditions associated with the corresponding impact events. Each experiment was separated into five smaller test segments to capture the profile evolution or around 1, 2, 4, 7 and 10 minutes. These segment durations were adjusted in response to the rapidity of the profile evolution during the early stages, and approaching equilibrium. The data acquisition rate was $100 \mathrm{~Hz}$, similar to the full-scale GWK tests. Figure 4 shows the experimental setup and instrumentation. 


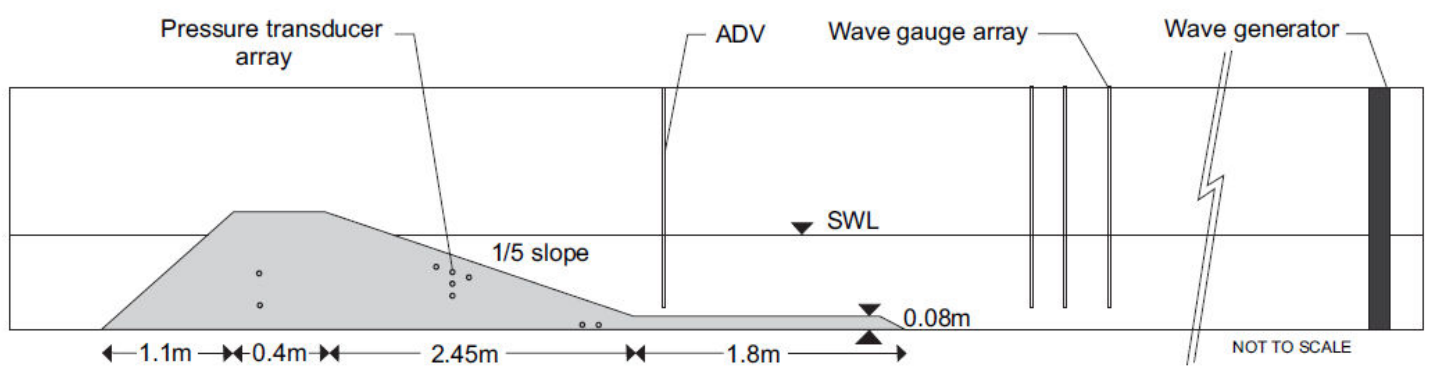

Figure 4. Experimental setup at UNAM

Figure 5 shows a typical view of the experimental beach at UNAM. The berm accretion can clearly be identified, as well as the excavated region below the initial profile line which is marked by the black tape on the tank wall.

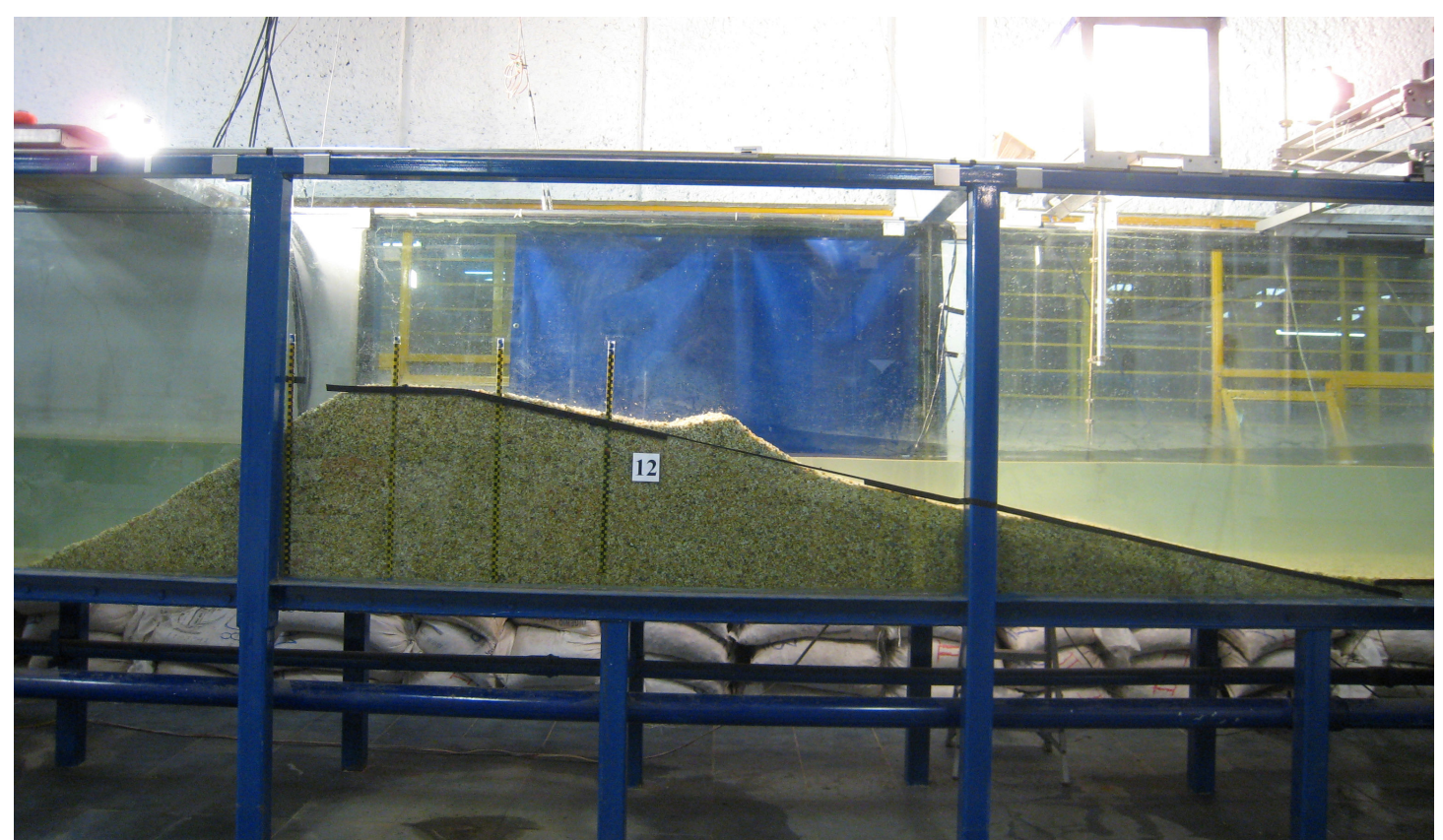

Figure 5. Cross-sectional view of the experimental beach at the UNAM facility

The data was pre-processed before analysis of the impact events could proceed, to account for shoaling, separation of reflected waves and depth attenuation of the pressure signal within the porous bed.

\section{Separation of incident and reflected waves}

The separation of the waves was carried out according to the methodology outlined in Frigaard and Brorsen (1995). This assumes that the recorded wave gauge data is a sum of an incident and reflected waves that are phase shifted from the original signal, and manipulation of the signals from two wave gauges can produce separation of incident and reflected waves with an accuracy comparable with other proposed methods.

\section{Wave shoaling}

Correction for shoaling was conducted following the empirical methodology presented in Goda (1975) for the calculation of the breaking wave height. Tsai et al. (2005) indicated that the application of this empirical methodology was in good agreement with practical experimentation for the more complicated wave height transformation that occurs on steep beaches such as the one included here.

\section{Pressure attenuation}

The pore pressure variations generated by oscillations in the water surface above a pressure transducer are attenuated through the beach medium with greater attenuation occurring the deeper the transducer is located within the beach. Pressure attenuation was accounted for through employment of the theory developed by Yamamoto et al. (1978), with utilisation of Massel et al. (2004) who presented 
expressions to allow for calculation of the wave number in a porous media. This is consistent with the approach adopted for the GWK experiments.

\section{Impact event processing}

In each record the impact events were identified, quantified and quality rated. The quality rating involved the assignment of a rating between five (best) and one (worst) according to the following criteria in Table 1:

\begin{tabular}{|c|l|}
\hline \multicolumn{2}{|c|}{ Table 1. Impact event quality scores. } \\
\hline Rating & Characteristics \\
\hline 5 & $\begin{array}{l}\text { Sharp distinct initial peak. Well-defined "church roof" profile. Distinct pressure } \\
\text { minimum. } \\
\text { Distinct initial peak. Well-defined "church roof" profile. Distinct pressure } \\
\text { minimum. Small level of noise may be present. } \\
\text { Initial pressure peak identifiable but less distinct. III-defined "church roof" profile. } \\
3\end{array} \quad \begin{array}{l}\text { Some noise present. } \\
\text { Indistinct maximum and/or minimum impact pressure. Ill-defined "church roof" } \\
\text { profile. Broad, indistinct initial pressure peak. Significant noise. } \\
\text { Indistinct maximum and/or minimum impact pressure. Ill-defined "church-roof" } \\
\text { profile and significant noise in the record. }\end{array}$ \\
\hline
\end{tabular}

Figure 6 shows example impact pressure responses for the various rating values (1-rated event not included as the signal is largely noise and contains no identifiable impact pressure spike).
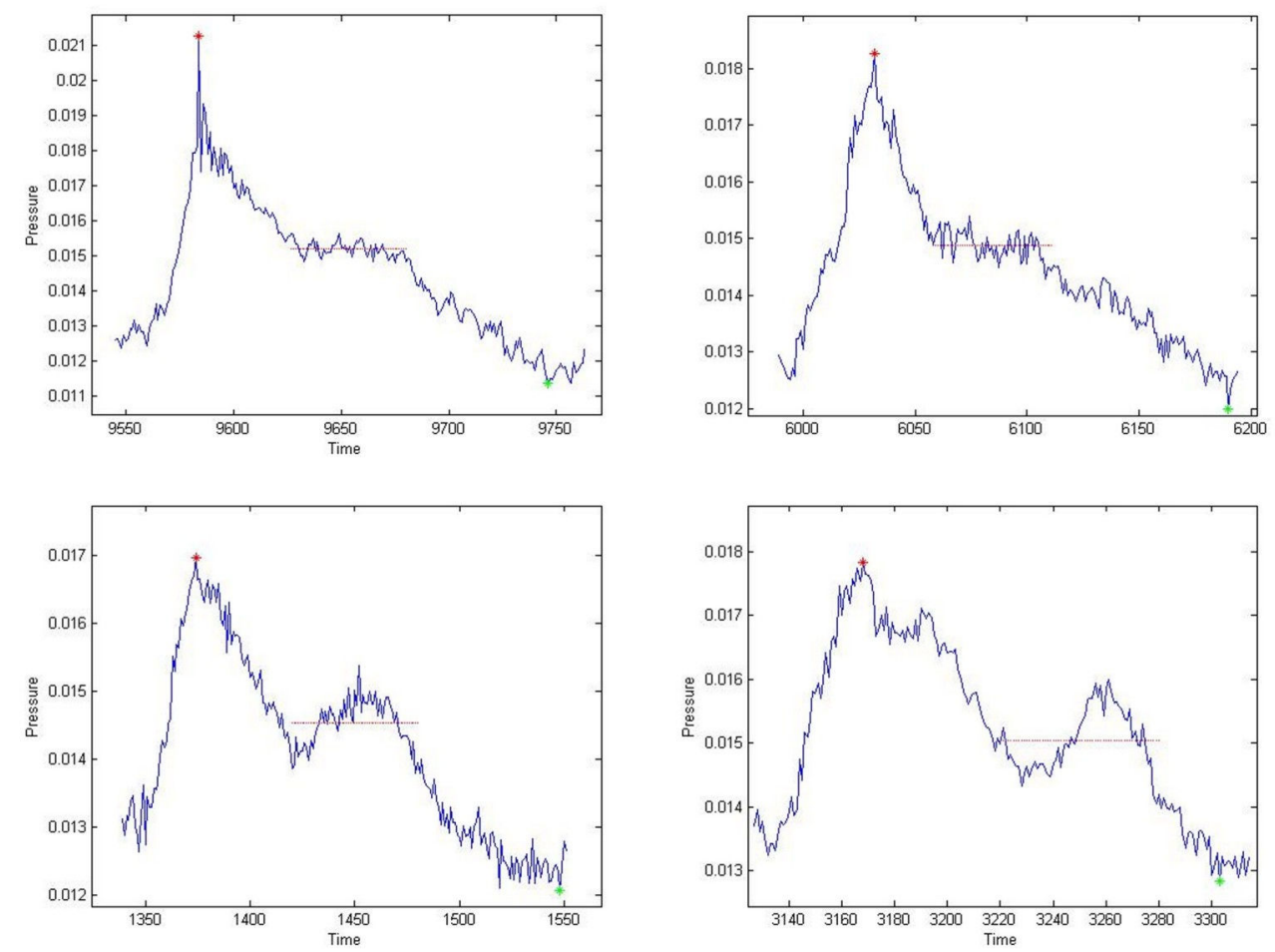

Figure 6. Example pressure response ratings, rated five (top left panel); rated four (top right panel); rated three (bottom left panel); and rated two (bottom right panel).

After each event was assessed, the results were filtered to include only impacts events rated as four or five. These events were included as there was a clear pressure spike relating to the initial plunging impact and the subsequent easily identifiable quasi-hydrostatic pressure, indicating the response is consistent with an impact close to the pressure transducer. The maximum and minimum pressures were extracted for each event and were taken forward for further analysis as described in the following section. 


\section{RESULTS}

Figure 7 presents the relationship between the non-dimensional impact pressure and the nondimensional wave height for the UNAM tests. There seems to be no discernable relationship between the variables, contrary to the observed monotonic relationship identified in the GWK data.

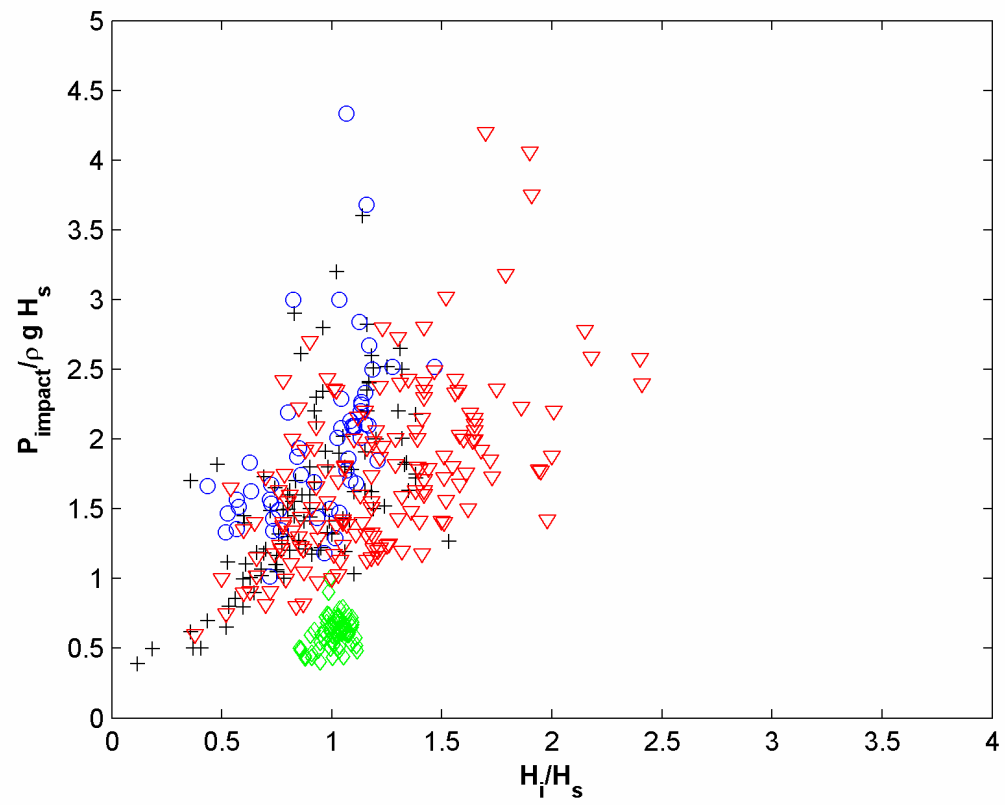

Figure 7. Non-dimensional impact pressure vs non-dimensional wave height for UNAM and GWK data (black cross $(+)$ blue circle $(\circ)$ and red triangle $(\nabla)$ - GWK data, green diamond $(\diamond)$ - UNAM data ) (using data from Pedrozo-Acuña et al., 2008)

The UNAM data can be seen to occupy a relatively narrow region of non-dimensional wave height $\left(0.8<\mathrm{H}_{\mathrm{i}} / \mathrm{H}_{\mathrm{s}}<1.2\right)$. This is much narrower than the GWK data shown, and the non-dimensional impacts appear to be smaller than the GWK data. This is likely due to the use of regular wave generation in the UNAM tests while the GWK tests utilised irregular wave generation.

Figure 8 presents the non-dimensional impact pressure $v s$ the surf-similarity parameter for UNAM and GWK data plotted together.

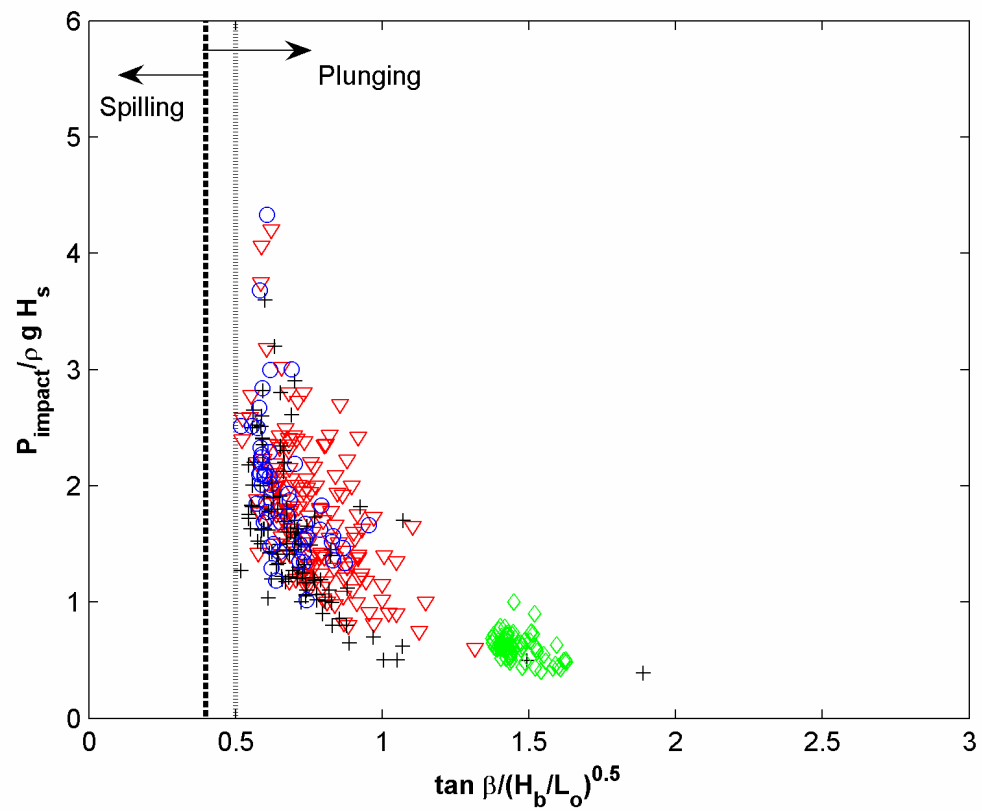

Figure 8. Non-dimensional impact pressure vs surf-similarity for UNAM and GWK data. (black cross (+) blue circle $(\circ)$ and red triangle ( $\nabla)$ - GWK data, green diamond $(\diamond)$ - UNAM data ) (using data from PedrozoAcuña et al., 2008) 
Whilst the new data is clustered over a narrow range of Iribarren values in the plunging region, it can be seen that it appears consistent with the broad trend in the Pedrozo-Acuna et al results. I.e., it indicates a continuation of the decline in the non-dimensional impact pressure as the surf-similarity parameter increases towards surging types.

\section{CONCLUSIONS}

This paper continues to build on the previous research which has indicated that the additional pressure induced by plunging breakers may play a significant role in the development of the morphological beach response (Pedrozo-Acuña, 2007).

New data for impacts on coarse grained beaches have been obtained at UNAM, at a different scale to the previous GWK tests. This additional data covers a region of the surf-similarity space previously untested. The apparent agreement between the UNAM and GWK (Figure 8) strengthens the hypothesis regarding the reduction in impacting intensity as wave breaking moves from plunging breakers towards surging breakers.

Larger data sets over a wide range of breaker types and scales are required. Particular attention should be paid to the interface between spilling and plunging wave breaking $\left(\xi_{b}=0.3-0.6\right)$ where there is currently a gap in this data and represents a region where an increase in non-dimensional impact pressure is expected as the surf-similarity parameter increases.

Additional parameterizations could also be attempted to also reduce the scatter. This might include an evaluation of the role of parameters such as roughness and permeability. Other preliminary tests have indicated that these further parameters can effect the relationship of the peak impact pressure and the Iribarren number. The ultimate goal of this work is to incorporate a parameterization into a description of the bed evolution in the plunging region of steep beaches.

\section{REFERNCES}

Bullock, G.N., Obhrai, C., Peregrine, D.H. and Bredmose, H. 2007. Violent breaking wave impacts. Part 1: Results from large-scale regular wave tests on vertical and sloping walls, Coastal Engineering, 54, 602-617.

Frigaard, P., and Brorsen, M., 1995. A time-domain method for separating incident and reflected irregular waves, Coastal Engineering, 24, 205-215.

Goda, Y., 1975. Irregular wave deformation in the surf zone. Coastal Engineering in Japan. 18, 13-26.

Jamal, M.H., Simmonds D.J., and Magar V., 2010. Modelling Infiltration on Gravel Beaches with an Xbeach Variant. Proceedings of $32^{\text {nd }}$ International Conference on Coastal Engineering, ASCE Submitted

Massel, S.R., Przyborska, A., and Przyborski, M., 2004. Attenuation of wave-induced groundwater pressure in shallow water. Part 1. Oceanologia, 46 (3) 383-404.

Mason, T., and Coates, T.T., 2001. Sediment transport processes on mixed beaches: a review for shoreline management. Journal of Coastal Research, 17 (3), 645-657.

Pedrozo-Acuña, A., Simmonds, D.J., Otta, A.K. and Chadwick, A.J. 2006. On the cross-shore profile change of gravel beaches, Coastal Engineering, 53, 335-347.

Pedrozo-Acuña, A., Simmonds, Chadwick, A.J., Silva-Casarin, R., 2007. A numerical-empirical approach for evaluating morphodynamic processes on gravel and mixed sand-gravel beaches, Marine Geology, 241, 1-18.

Pedrozo-Acuña, A., Simmonds, D.J. and Reeve, D.E., 2008. Wave-impact characteristics of plunging breakers on gravel beaches, Marine Geology, 253, 26-35.

Tsai, C.-P., Chen, H.-B., Hwung, H.-H., and Huang M.-J., 2005. Examination of empirical formulas for wave shoaling and breaking on steep slopes, Ocean Engineering, 32, 469-483. 